\title{
Suicide : Leading Cause of Death among Women in Nepal
}

\section{Karki C}

Kathmandu Medical College

Sinamangal, Kathmandu.

World Health Organization (WHO) reports that suicide is a leading cause of death among people under 25 and about one million people commit suicide every year which comes to around 3,000 deaths a day or one death every 40 seconds and $60 \%$ of all suicide cases occur in Asia. ${ }^{1}$ Even though more men commit suicide, more women attempt to do so.

Risk factors for suicide vary in different cultures. Mental disorders like depression or alcohol and substance abuse play a major role in Western society where as impulsiveness plays a much more important role in Asian countries implying that suicide can happen on the spur of a moment in an otherwise unsuspecting individual. $^{2}$

An epidemiologic review of suicide and its prevention was done in Canada in the year 1989. The risk factors found were age- men aged 70 years or above, women aged 65 to 69 and men aged 20 to 24 , mental illness, previous suicidal attempt, life-threatening illness, native people, people with a family history of suicide and prisoners in the descending order. ${ }^{3}$ Unlike the findings in Canada, suicidal death in Nepal is more common among women of reproductive age group. ${ }^{4} \mathrm{It}$ is in fact the leading cause of death among women of reproductive age in Nepal probably due to domestic violence from their husband and in laws as over eighty percent of them report experiencing domestic violence. ${ }^{5}$

A review of evidence: Suicide among women in Nepal was published following wide discussion among Safe Motherhood Network Federation involving various stakeholders like UNFPA, Nepal Police, Ministry of Health and Population and others during its $3^{\text {rd }}$ international conference on safe motherhood. The report confirms that suicide is a major latent public health ailment among women of reproductive age group in Nepal. ${ }^{6}$

Maternal Mortality Ratio (MMR) study of 2008/2009 and the recently released report on the review of the evidences on suicidal tendencies among women in Nepal have clearly highlighted that suicide among women in Nepal is on rise. ${ }^{7}$

The Maternal Mortality and Morbidity Study 2008/2009 undertaken by the Family Health Division (FHD) of the Department of Health Services of Nepal looked at changes in maternal mortality in Nepal since 1998. This study covering a population of over 86,000 women of reproductive age group was carried out in eight districts chosen to represent different ethnicities and levels of development in Nepal which concluded that even though overall maternal mortality rate in Nepal improved to 229 from 539 maternal deaths per 100,000 live births in 1998, suicide (16\%) rather than maternal related issues (12\%) was the single leading cause of death in that age group which was higher than similar report of $1898(10 \%)$ in which suicide was third leading cause of death. ${ }^{7}$

It clearly indicates that suicidal deaths in this age group are actually in the rise. Poisoning, mainly ingestion of pesticides, was the most common means of committing suicide. ${ }^{8}$ Considering the scale of domestic violence experienced by women, results were not surprising, and that the problem could be even more widespread as suicides are under-reported due to fear of being entangled in legal problems. There is a need to understand the story behind the maternal mortality change over ten years and to explore and identify contributory causes and their relative importance and to put in place necessary steps to prevent maternal deaths in future.

Every suicide is a cry for help; an unmet need for urgent intervention for a woman who is trapped in a situation where she has reached the limit of her capacity to cope with violence, abuse and discrimination. Maternal health care delivery package focuses on physical care in terms of antenatal, natal and postnatal care and does not give much importance to mental health. Prevention of suicide is a complex task and it requires understanding of social, economic and cultural issues as well. This issue has to be addressed urgently.

\section{REFERENCES}

1. Government of Nepal, Ministry of Health and Population, Department of Health services, Kathmandu. Annual Report Department of Health services 2066-2067 (2009-2010). 
2. Patel V, Kleinman A (2003). Poverty and common mental disorders in developing countries. Bulletin of World Health Organization. 81(8): 609-15.

3. Jane E. Mcnamee, MA; David R. Offord, MD. Prevention of suicide. Can Med Assoc J 1990;142(11).

4. Pathak L R, Malla D S, Pradha. A et al. Maternal mortality and morbidity study. 1998. Family Health Division, Department of Health services, Ministry of Health, His Majesty's Government of Nepal.

5. Joshi S K . Violence against women in Nepal: Role of Health care workers. Kathmandu university medical journal 2009;7(2):89-91.

6. Pradhan A, Poudel P, Thomas D, Barnett S. A review of the evidence: suicide among women in Nepal. July 2011. Options consultancy services Ltd, UKaid and UNFPA.

7. Pradhan A, Suvedi B K, Barnett S et al. Nepal Maternal mortality and morbidity study 2008/2009.Family Health Division, Department of Health services, Ministry of Health, His Majesty's Government of Nepal.

8. Ajdacic- Gross, $V$ Weiss, M G Ring et al. Methods of suicide: international suicide patterns derived from the WHO mortality database. Bulletin of World Health Organisation 86(9): 657-736 